\title{
Differential expression of neuregulin-1 isoforms and downregulation of erbin are associated with Erb B2 receptor activation in diabetic peripheral neuropathy
}

Pan Pan and Rick T Dobrowsky

\begin{abstract}
Background: Aberrant neuron/glia interactions can contribute to a variety of neurodegenerative diseases and we have previously demonstrated that enhanced activation of Erb B2, which is a member of the epidermal growth factor receptor (EGFR) family, can contribute to the development of diabetic peripheral neuropathy (DPN). In peripheral nerves, Erb B receptors are activated by various members of the neuregulin-1 (NRG1) family including NRG1 Type I, NRG1 Type II and NRG1 Type III to regulate Schwann cell (SC) growth, migration, differentiation and dedifferentiation. Alternatively, Erb B2 activity can be negatively regulated by association with the Erb B2-interacting protein, erbin. Since the effect of diabetes on the expression of NRG1 isoforms and erbin in peripheral nerve are unknown, the current study determined whether changes in NRG1 isoforms and erbin may be associated with altered Erb B2 signaling in DPN.
\end{abstract}

Results: Swiss Webster mice were rendered diabetic with streptozotocin (STZ) and after 12 weeks of diabetes, treated with erlotinib, an inhibitor of Erb B2 activation. Inhibition of Erb B2 signaling partially reversed several pathophysiologic aspects of DPN including a pronounced sensory hypoalgesia, nerve conduction velocity deficits and the decrease in epidermal nerve fiber innervation. We also observed a decrease of NRG1 Type III but an increase of NRG1 Type I level in diabetic sural nerves at early stage of diabetes. With disease progression, we detected reduced erbin expression and enhanced MAPK pathway activity in diabetic mice. Inhibition of Erb B2 receptor suppressed MAPK pathway activity in treated-diabetic sural nerves.

Conclusions: These results support that hyperglycemia may impair NRG1/Erb B2 signaling by disrupting the balance between NRG1 isoforms, decreasing the expression of erbin and correspondingly activating the MAPK pathway. Together, imbalanced NRG1 isoforms and downregulated erbin may contribute to the dysregulation of Erb B2 signaling in the development of DPN.

Keywords: Nerve conduction velocity, Erlotinib, Sensory hypoalgesia, Intra epidermal nerve fibers, Sural nerve

\footnotetext{
* Correspondence: dobrowsky@ku.edu

Department of Pharmacology and Toxicology, University of Kansas,

Lawrence, KS, USA
}

\section{Biomed Central}

(c) 2013 Pan and Dobrowsky; licensee BioMed Central Ltd. This is an Open Access article distributed under the terms of the Creative Commons Attribution License (http://creativecommons.org/licenses/by/2.0), which permits unrestricted use, distribution, and reproduction in any medium, provided the original work is properly cited. 


\section{Background}

Diabetic peripheral neuropathy (DPN) is one of the most prevalent complications of chronic diabetes and is the leading cause of non-traumatic lower limb amputation [1]. DPN patients often present with spontaneous positive (burning, itching, abnormal sensation to temperature, pain) or negative (numbness, insensitivity) sensory symptoms that are observed in a distal-to-proximal manner [2]. DPN patients often exhibit a reduction in nerve conduction velocity, loss of nerve fibers and segmental demyelination [2,3]. Mechanistically, the development of DPN has multiple contributing factors. Hyperglycemia induces oxidative stress and alters several metabolic and vascular pathways that contribute to an increase in formation of advanced glycation end-products, excessive polyol synthesis, enhanced protein kinase $\mathrm{C}$ activation and an increase in poly-ADP ribose polymerase activity [4]. Another potential mechanism is altered neurotrophism since hyperglycemia can damage peripheral nerves by changing the expression, proteolytic cleavage, axonal transport and function of growth factors. For instance, diabetic rats showed decreased retrograde axonal transport of nerve growth factor (NGF) and brain-derived neurotrophic factor (BDNF), members of the neurotrophin family [5]. In addition, insulin-like growth factors affect neuronal survival, growth and regeneration and are decreased in nerve of diabetic rats [6]. Neuregulin 1 (NRG1) is a growth factor that interacts with Erb B receptors, which localize primarily to Schwann cells (SC), and is critical for both SC differentiation and degeneration. However, little is known about how diabetes may affect gliotrophic signaling by altering NRG1 levels and Erb B2 signaling in the peripheral nervous system (PNS).

The NRG1 family comprises more than 30 membranebound and secreted proteins generated by alternative promoter usage and extensive RNA splicing [7]. The NRG1 family is subdivided into six major isoforms based on distinct amino termini but NRG1 Types I, II, and III are the best characterized in the peripheral nervous system. However, all NRG1 family members have an epidermal growth factor (EGF)-like domain which is sufficient and necessary to activate the Erb B members of the EGF receptor (EGFR) family [8]. The EGFR family contains four members, EGFR, Erb B2, Erb B3 and Erb B4. Erb B2 is a ligand-less receptor and Erb B3 has no tyrosine kinase activity. In adult SCs, NRG1 binding to Erb B3 induces formation of primarily Erb B2 and Erb B3 heterodimers [9]. Receptor dimerization activates the intrinsic tyrosine kinase activity of Erb B2 which initiates downstream signaling pathways.

NRG1/Erb B signaling guides every developmental stage of the SC lineage, such as promoting the gliogenic fate of neural crest cells, migration of SC precursors and subsequent proliferation, survival, and differentiation
$[8,10]$. Physiologically, the axonal expression of NRG1 Type III is necessary to promote myelination and regulate myelin thickness in vivo [11,12]. Moreover, disruption of Erb B signaling by transgenic overexpression of a dominant-negative Erb B4 receptor resulted in abnormal myelin formation, disrupted Remak bundle structure, and neuropathic changes in both myelinated [13] and unmyelinated [14] sensory fibers. Interestingly, pathologic activation of Erb B2 following axotomy can also promote SC dedifferentiation and demyelination [15]. Though NRG1 Type III promotes myelination, NRG1 Type I and NRG1 Type II have been shown to induce demyelination in vitro [16] and this dedifferentiation response is tightly linked to the activation of the $\mathrm{p} 42 / \mathrm{p} 44$ MAPK pathway $[17,18]$. Therefore, changes in the expression of isoforms of NRG1 may provide one mechanism for activation of Erb B2 and p42/p44 MAPK signaling in pathophysiologic states linked to neuropathy and myelin degeneration. Furthermore, Erb B2 is also negatively regulated by the Erb B2-interacting protein (erbin), a downstream adapter protein that specifically interacts with Erb B2, but not Erb B3 or Erb B4 [19]. Erbin is mostly expressed in regions where myelination is abundant and erbin null mice have hypomyelinated peripheral nerves, suggesting it is necessary for normal myelin thickness of peripheral nerves [20]. Following recruitment to Erb B2, erbin can inhibit the p42/p44 MAPK pathway and potentially antagonize NRG1-induced demyelination.

We have previously demonstrated that a transient increase in Erb B2 receptor activation contributed to diabetes-induced sensory neuropathy since inhibiting Erb B2 signaling reversed some of the pathophysiologic features of DPN [21]. Therefore, disrupted Erb B2 signaling might contribute to the axonal degeneration and $\mathrm{SC}$ demyelination in diabetic nerves. However, it remains unknown whether the increase in Erb B2 activity in diabetic nerves is associated with changes in NRG1 isoforms and erbin that may serve as positive and negative regulators of Erb B2 signaling, respectively.

To elucidate the role of altered NRG1/Erb B2 signaling in DPN, we used diabetic Swiss Webster mice, which after prolonged diabetes develop severe pathophysiologic symptoms of DPN [22]. We report that diabetes altered the expression of NRG1 Type III and NRG1 Type I in distal nerve fibers with increasing duration of diabetes. In particular, a decrease in NRG1 Type III expression was accompanied by an increased expression of NRG1 Type I level in diabetic sural nerves. Additionally, the levels of erbin were decreased in sciatic nerves of diabetic mice and this corresponded with an increase in p42/p44 MAPK pathway activity. These data are the first to characterize that diabetes can alter the expression of proteins that serve as positive and negative regulators of 
Erb B2 activity and suggest that an altered neuregulinism may contribute to SC pathology in DPN.

\section{Results}

Erb B2 receptor inhibitor attenuates pathophysiological indices of DPN

Swiss Webster mice were rendered diabetic with STZ and changes in mechanical and thermal sensitivity were monitored for 12 weeks to assess the onset of hypoalgesia in both myelinated (mechanical) and unmyelinated (thermal) nerve fibers. Diabetes induced a clear mechanical (Figure 1A) and thermal (Figure 1B) hypoalgesia and after 12 weeks this was associated with a significant decrease in both motor (MNCV) and sensory (SNCV) nerve conduction velocity compared to non-diabetic animals (Figure $1 \mathrm{C}$ and D). To address whether Erb B2 signaling contributed to the sensory deficits, mice were treated at week 13 with vehicle or $25 \mathrm{mg} / \mathrm{kg}$ erlotinib, which is a clinically approved inhibitor of the EGFR that can also inhibit Erb B2 receptors [21]. The mice were initially given one treatment per week for 4 weeks and this modestly improved mechanical and thermal sensitivity. Increasing the frequency of dosing to twice per week (Figure 1A and B, dashed arrows) yielded a greater improvement in mechanical sensitivity, but thermal sensitivity did not change beyond that seen with a single dose per week. After 8 weeks of erlotinib treatment, the pre-existing deficits in both MNCV and SNCV were partially reversed (Figure $1 C$ and D). Interestingly, the improvement in SNCV and thermal hypoalgesia in erlotinib-treated diabetic Swiss Webster mice was not observed in our previous study using $\mathrm{C} 57 \mathrm{Bl} / 6$ mice [21]. Whether this observation is attributable to differences between the mouse strains is unclear. Nonetheless, these data support that Erb B2 signaling may contribute to glial cell dysfunction in DPN.

Assessing changes in intraepidermal nerve fiber density (IENFD) is emerging as a powerful technique for accurately diagnosing and staging the onset of a small fiber
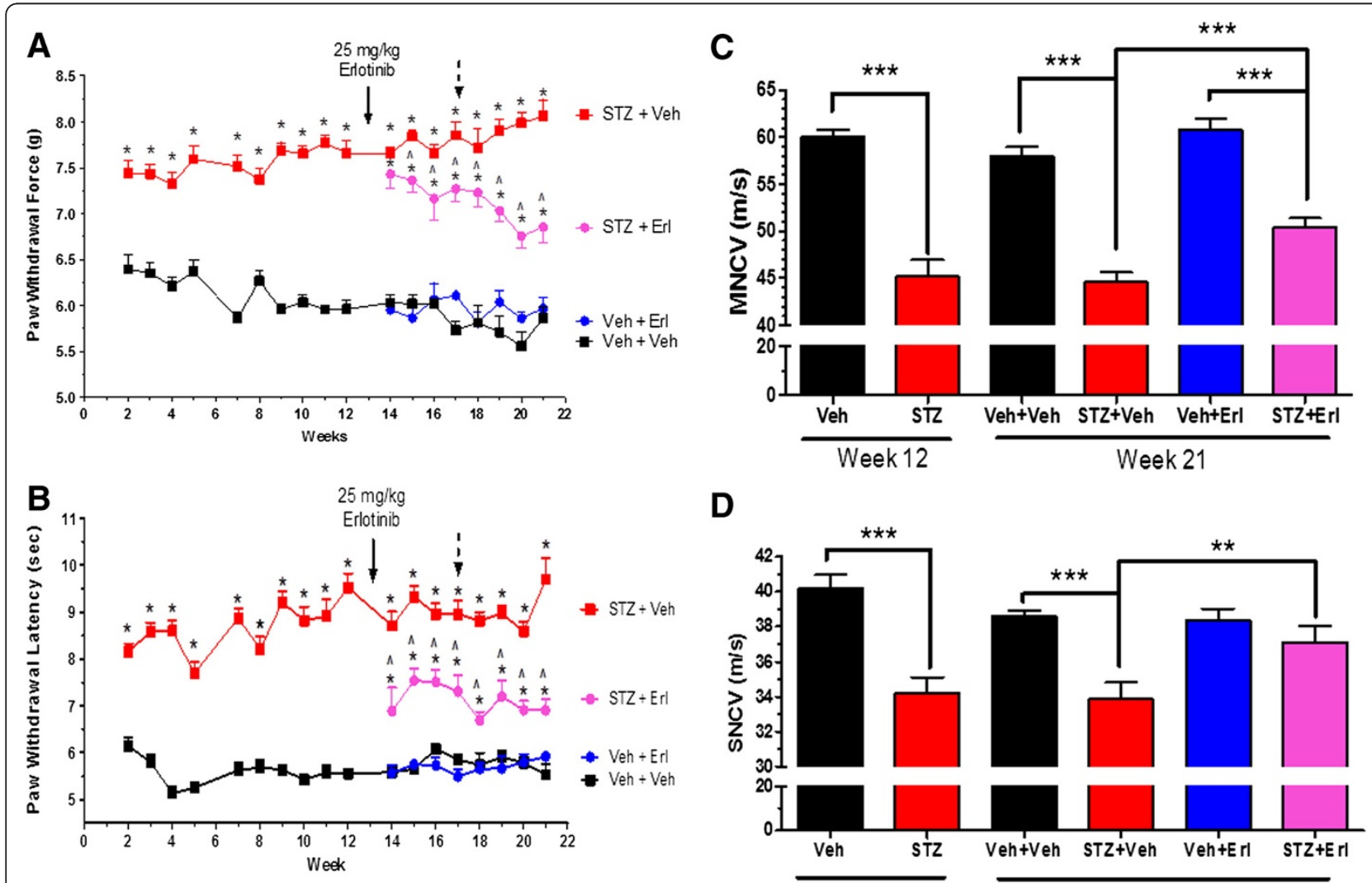

D

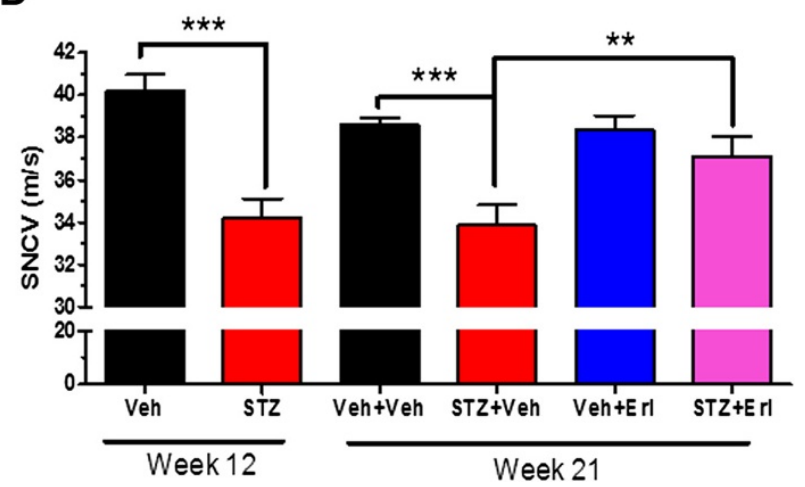

Figure 1 Inhibition of Erb B2 attenuated NCV and sensory deficits in diabetic mice. Swiss Webster mice were rendered diabetic and after two weeks of diabetes, mechanical (A) and thermal sensitivity (B) were assessed weekly $\left(^{*}, p<0.05\right.$ versus time-matched Veh + Veh; $\wedge$, $p<0.05$ versus time-matched STZ + Veh). Assessment of MNCV (C) and SNCV (D) in a subgroup of animals ( $\mathrm{n}=4$ per group) after 12 weeks of diabetes confirmed the onset of nerve dysfunction prior to drug treatment. At 13 weeks of diabetes, animals were given vehicle or 25 mg/kg erlotinib once per week for 4 weeks (solid arrow) and then twice per week (dashed arrow) for the final four weeks ( $n=8-12$ per group). Erlotinib significantly improved mechanical and thermal sensitivity and decreased the deficits in both MNCV and SNCV compared to vehicle treated diabetic mice $\left(* *, p<0.01 ;{ }^{* * *}, p<0.001\right)$. 
neuropathy in patients [23] and animal models [24,25]. We processed the plantar surface of the hind paws and stained the nerve fibers with an antibody against protein gene product 9.5 (PGP 9.5), which is a cytosolic ubiquitin C-terminal hydroxylase specifically expressed in neurons. The PGP 9.5-positive nerve fibers that crossed the epidermal/dermal junction were counted to quantify distal nerve innervation (Figure 2A and B). As anticipated, 12 weeks of diabetes significantly decreased IENFD but fiber loss was not significantly greater after 21 weeks of diabetes. However, erlotinib treatment partially reversed the diabetes-induced loss of the largely unmyelinated intra-epidermal nerve fibers. Though the increase in IENFD in erlotinib-treated diabetic mice may contribute to the increased sensitivity to noxious thermal stimuli, improved thermal sensitivity can occur in the absence of an increase in epidermal fibers $[25,26]$.

After 12 and 21 weeks of diabetes, fasting blood glucose (FBG) levels and HbA1c were increased. As expected, diabetic mice showed a loss of body weight
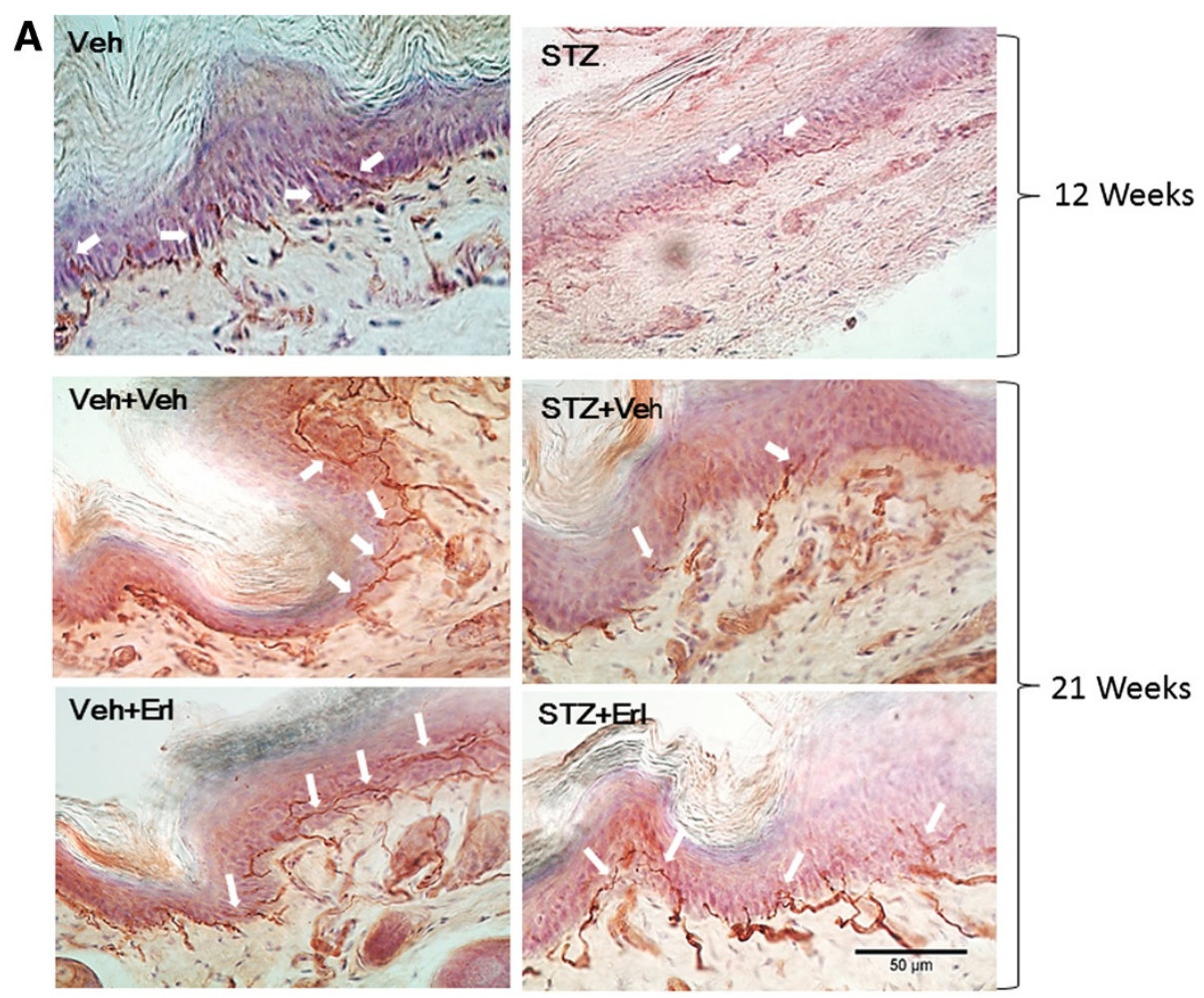

-21 Weeks

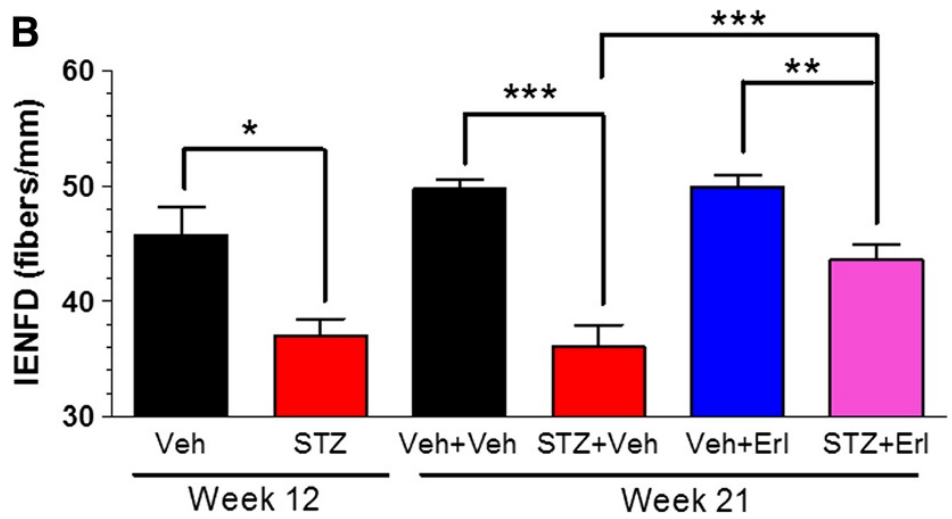

Figure 2 Inhibition of Erb B2 improved IENFD in DPN mice. Footpad samples were collected from the plantar surface of the hind paws after 12 ( $n=4$ per group) or 21 weeks ( $n=6-8$ per group) of treatment. (A): Representative images of IENFD in non-diabetic (Veh) and diabetic (STZ) mice treated with vehicle (Veh) or erlotinib (Erl). Nerve fibers immunopositive for PGP 9.5 are stained red by the chromagen (arrows) and epidermal cells were stained purple by hematoxylin. (B): Quantification demonstrated a significant loss of nerve innervation in diabetic mice prior to drug administration and erlotinib treatment induced a partial recovery in fiber loss $\left({ }^{*}, p<0.05 ;{ }^{* *}, p<0.01{ }^{* * *}, p<0.001\right)$. 
compared to age-matched, vehicle-treated control mice (Table 1) and erlotinib treatment did not affect FBG or body weight compared to vehicle-treated diabetic mice. These results indicate that the effect of erlotinib on improving the diabetes-induced nerve deficits in both myelinated and unmyelinated nerve fibers were not related to correction of the systemic hyperglycemia, consistent with our previous observation in diabetic $\mathrm{C} 57 \mathrm{Bl} / 6$ mice [21].

\section{Diabetes differentially alters the expression of NRG1 isoforms in sural nerve}

Though Erb B2 activity can contribute to DPN, it is unknown whether this may be associated with changes in the expression of NRG1 isoforms which can promote SC differentiation or dedifferentiation. Sciatic and sural nerves were isolated from control and diabetic mice, protein lysates were separated by SDS-PAGE and probed with two different NRG1 Type III antibodies. Using a previously reported strategy [12], we raised a polyclonal antibody against a peptide sequence near the distinct cysteine-rich domain (CRD) in the NRG1 Type III isoform. Immunoblot of sciatic nerve extracts with this antibody detected a prominent band at about $75 \mathrm{kDa}$ (Figure 3A). This band represents the N-terminal fragment after cleavage and is consistent with the protein size previously reported [27]. As a complementary approach, we also used a well characterized commercial NRG1 Type III antibody that was raised against a region within the C-terminal domain of NRG1 Type III (SMDF, sensory and motor neuron derived factor) and detects a band at about $65 \mathrm{kDa}$.

To confirm antibody specificity, $0.5 \mu \mathrm{g} / \mathrm{ml}$ of the NRG1 Type III antibody was preabsorbed with the immunizing peptide in a $1: 50$ to $1: 200$ mass ratio prior to immunoblot analysis of sciatic nerve lysates (Figure 3B). The immunizing peptide decreased the amount of NRG1 Type III present at $75 \mathrm{kDa}$ but had no effect on the band present at $50 \mathrm{kDa}$, indicating it is nonspecifically recognized by the antibody. Though the identity of the minor $37 \mathrm{kDa}$ band in Figure $3 \mathrm{~A}$ is

Table 1 Body weights, FBG and HbA1c values from the Swiss Webster mice

\begin{tabular}{ccccccc}
\hline Week & Treatment & FBG (mg/dl) & Weight (g) & (n) & HbA1c (\%) & (n) \\
\hline Week 12 & Veh & $112 \pm 21$ & $37.2 \pm 5.4$ & 10 & $5.0 \pm 0.4$ & 6 \\
& STZ & $595 \pm 12^{*}$ & $30.6 \pm 3.7^{*}$ & 11 & $12.9 \pm 0.2^{*}$ & 7 \\
Week 21 & Veh + Veh & $121 \pm 26$ & $42.6 \pm 5.4$ & 8 & $5.1 \pm 0.1$ & 3 \\
& Veh + Erl & $104 \pm 17$ & $43.4 \pm 2.7$ & 8 & $5.0 \pm 0.2$ & 3 \\
& STZ + Veh & $545 \pm 106^{*}$ & $34.5 \pm 3.5^{*}$ & 8 & $10.2 \pm 1.7^{*}$ & 4 \\
& STZ + Erl & $584 \pm 40^{\#}$ & $37.0 \pm 5.5^{\#}$ & 6 & $11.4 \pm 1.1^{\#}$ & 4 \\
\hline
\end{tabular}

${ }^{*}, \mathrm{p}<0.05$ vs. Veh or Veh + Veh; \#, $\mathrm{p}<0.05$ vs. Veh + Erlotinib (Erl). unknown, it may represent a degradation product of NRG1 Type III as it was not consistently detected. Importantly, preabsorbing the peptide to an antibody against NRG1 Type I (Figure 3C) or the NRG1 Type III antibody that was raised against a region within the $C$ terminal domain of NRG1 Type III (Figure 3D) did not decrease the detection of these NRG1 isoforms, further supporting the specificity for the custom antibody against an N-terminal fragment of NRG1 Type III. Immunoblotting for $\beta$-actin indicated that similar amounts of protein were present in each sample. Unfortunately, we were unable to detect the presence of the $165 \mathrm{kDa}$ unprocessed NRG1 with any antibodies used in the current work.

After 9 weeks (Figure 4A) and 12 weeks (Figure 4B) of diabetes, immunoblot analysis with both antibodies showed a significant decrease in the $75 \mathrm{kDa}$ and $65 \mathrm{kDa}$ NRG1 Type III bands in sural nerve (Figure 3D). Similarly, the expression of NRG1 Type III was also decreased in sciatic nerve after 9 and 12 weeks of diabetes (Figure 5A-C). Given the role of axonal NRG1 Type III in promoting $\mathrm{SC}$ differentiation, these data suggest that diabetes may compromise myelination by decreasing NRG1 Type III levels. However, a decrease in NRG1 Type III would not be anticipated to stimulate Erb B2 activity in peripheral nerve. Since NRG1 Type I can stimulate SC dedifferentiation [17], we also investigated whether altered expression of this isoform is associated with DPN. After 9 weeks of diabetes, the level of NRG1 Type I was modestly increased in sural nerve but this response was more robust after 12 weeks (Figure 6A and B). However, the levels of NRG1 Type I in diabetic sciatic nerve remained unchanged at both time points (data not shown). These data suggest that diabetes can differentially alter the expression of NRG1 isoforms, especially in the largely sensory sural nerve.

\section{Diabetes decreases erbin expression and increases p42/ p44 MAPK activity}

Erbin is an Erb B2 interacting protein that can function as a negative regulator of receptor signaling, in part by inhibiting p42/p44 MAPK activity [28]. Thus, a decrease in erbin expression may also contribute to potential dysregulation of Erb B2 signaling in diabetic nerve. Diabetic sciatic nerve showed a decreased expression of erbin at 12 and 16 weeks (Figure 7A-D). However, enhanced activity of $\mathrm{p} 42 / \mathrm{p} 44$ MAPK pathway was only detected in 16-week diabetic sciatic nerves (Figure 7D). Though it was difficult to detect erbin in sural nerve, its level also decreased after 21 weeks of diabetes and this correlated with an increase in phosphorylation of $\mathrm{p} 42 /$ p44 MAPK. Treatment with erlotinib treatment significantly decreased the extent of MAPK phosphorylation (Figure 8A and B). 


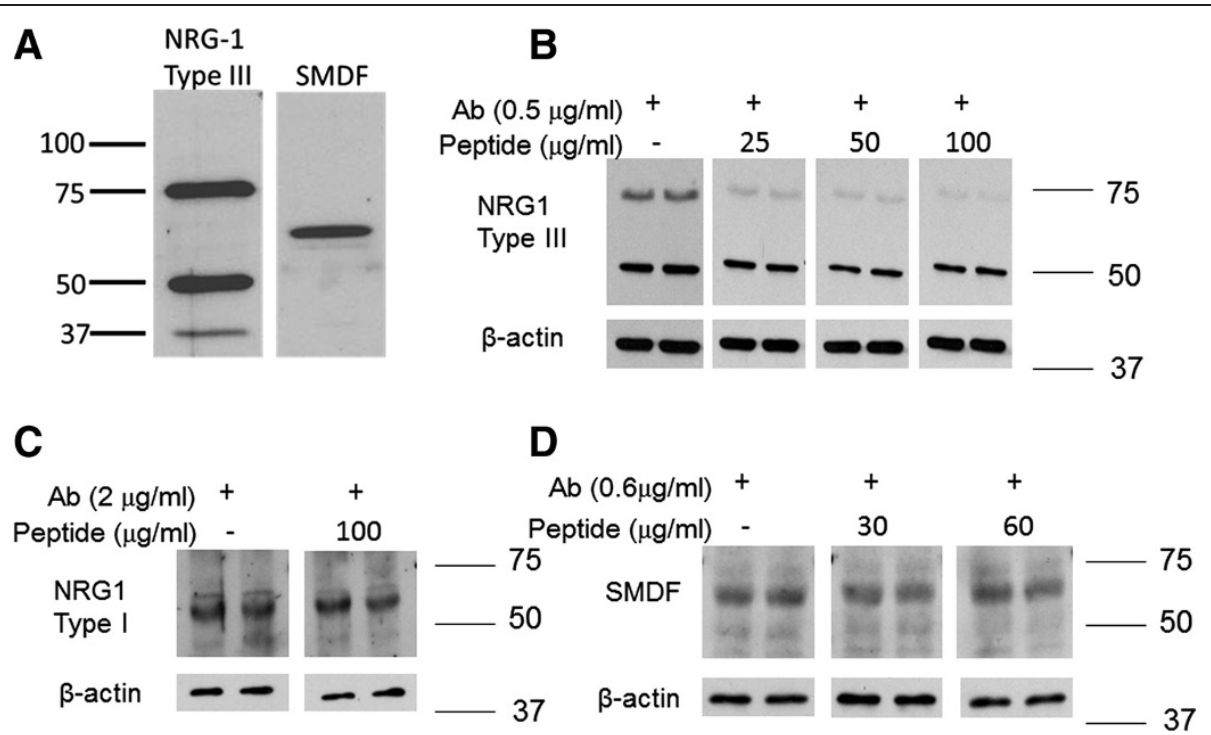

Figure 3 Validation of specificity of the custom NRG1 Type III antibody. (A): Comparison of NRG1 Type III detection using the custom N-terminal NRG1 Type III polyclonal antibody (left) and a commercial C-terminal NRG1 Type III polyclonal antibody (SMDF, right). (B-D) Demonstration of the specificity of the N-terminal NRG1 Type III polyclonal antibody. The indicated amounts of the N-terminal NRG1 Type III antibody (B), a polyclonal antibody against NRG1 Type I (C) or the C-terminal NRG1 Type III antibody (SMDF, D) were preabsorbed with the immunizing peptide in a 1:50 to 1:200 ratio for $1 \mathrm{hr}$ at $25^{\circ} \mathrm{C}$ and then used for immunoblot analysis of a sciatic nerve sample. The peptide specifically decreased the detection of the 75 kDa NRG1 Type III band but had no effect on detecting NRG1 Type I or the 65 kDa C-terminal NRG1 Type III fragment.

\section{Discussion}

Our prior work was the first to suggest that altered signaling via Erb B2 can contribute to the onset of DPN [21] but it was unclear if changes in Erb B2 signaling may be associated with an altered expression of NRG1.
The current study extends this work and is the first to demonstrate that diabetes can alter the expression of NRG1 isoforms in peripheral nerves. While we observed a reduction in NRG1 Type III levels in both diabetic sciatic and sural nerves, the level of NRG1 Type I increased

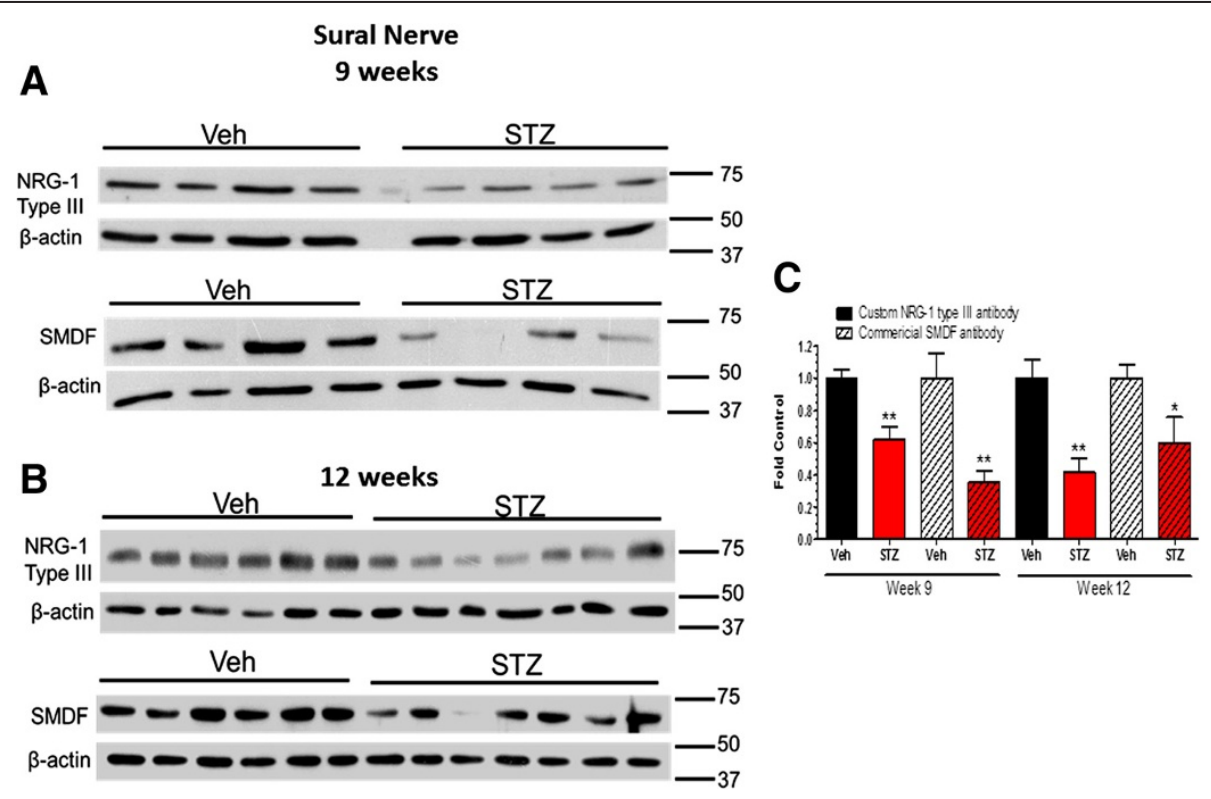

Figure 4 NRG1 Type III was decreased in sural nerve after 9 and 12 weeks of diabetes. Mice were treated with vehicle (Veh) or STZ and sural nerves were isolated at 9 (A) or 12 (B) weeks after the induction of diabetes ( $n=6-7$ per group). Protein lysates were prepared and NRG1 Type III level was determined by immunoblot using the two antibodies. (C): Bands were quantified, NRG1 Type III levels were normalized to $\beta$-actin and expressed as a fold of the levels in control sural nerves ( ${ }^{*}, p<0.05$ versus Veh; ${ }^{* *}, p<0.01$ versus Veh). 

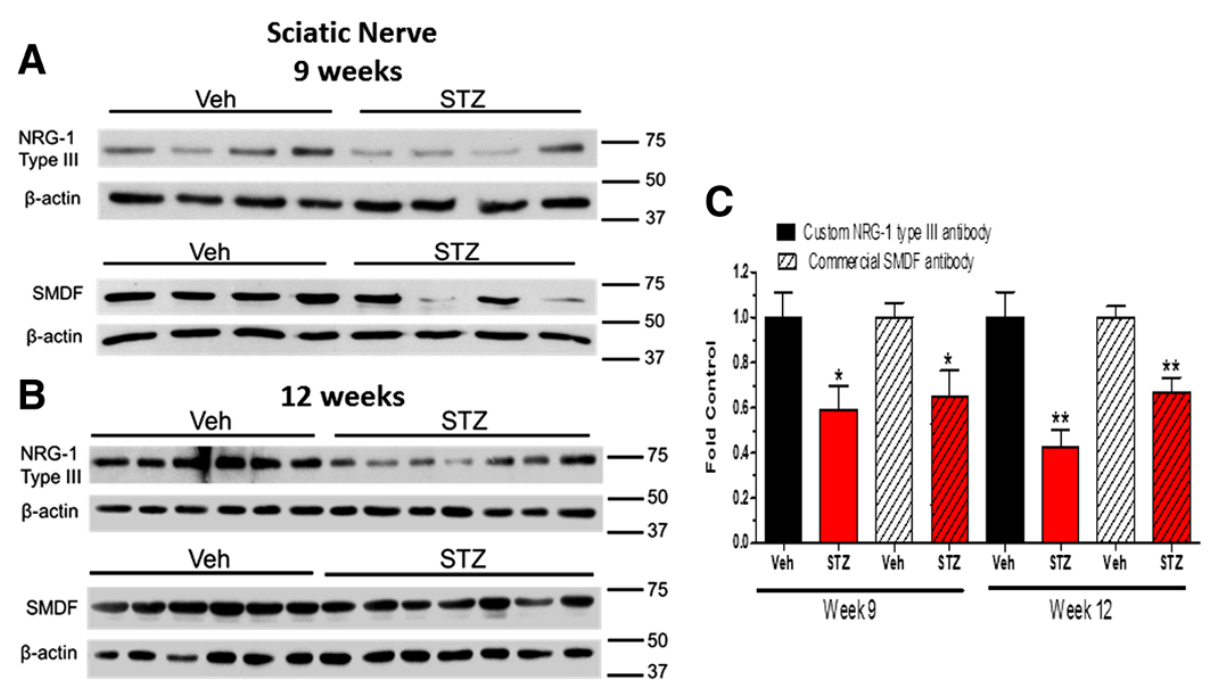

Figure 5 NRG1 Type III was decreased in sciatic nerve after 9 and 12 weeks of diabetes. Mice were treated with vehicle (Veh) or STZ and sciatic nerves were isolated at 9 (A) or 12 (B) weeks after the induction of diabetes ( $n=6-7$ per group). Protein lysates were prepared and NRG1 Type III level was determined by immunoblot using the two antibodies. (C): Bands were quantified, NRG1 Type III levels were normalized to $\beta$-actin and expressed as a fold of the levels in control sciatic nerves ( ${ }^{*}, p<0.05$ versus Veh; ${ }^{* *}, p<0.01$ versus Veh).

in sural nerve after 9-12 weeks of diabetes. Since inhibition of Erb B2 with erlotinib partially reversed the sensory deficits associated with prolonged diabetes, the enhanced expression of NRG1 Type I in diabetic sural nerves might be sufficient to elevate Erb B2 receptor activation and contribute to the progression of DPN. Moreover, the decreased NRG1 Type III and increased NRG1 Type I expression observed in diabetic sural nerve (but not in diabetic sciatic nerves) implies that distal and more thinly myelinated fibers may be more sensitive to early disruption of NRG1 signaling. This would be consistent with recent results showing a greater severity of oxidative stress in distal sural nerve compared to sciatic nerve [29].

NRG1 Type III is a membrane-anchored precursor protein which needs to be proteolytically processed to become an active signaling molecule. The $\beta$-secretase BACE1 ( $\beta$-site amyloid precursor protein cleaving enzyme 1 ) and the $\alpha$-secretase TACE (tumor necrosis factor- $\alpha$-converting enzyme) are two enzymes that

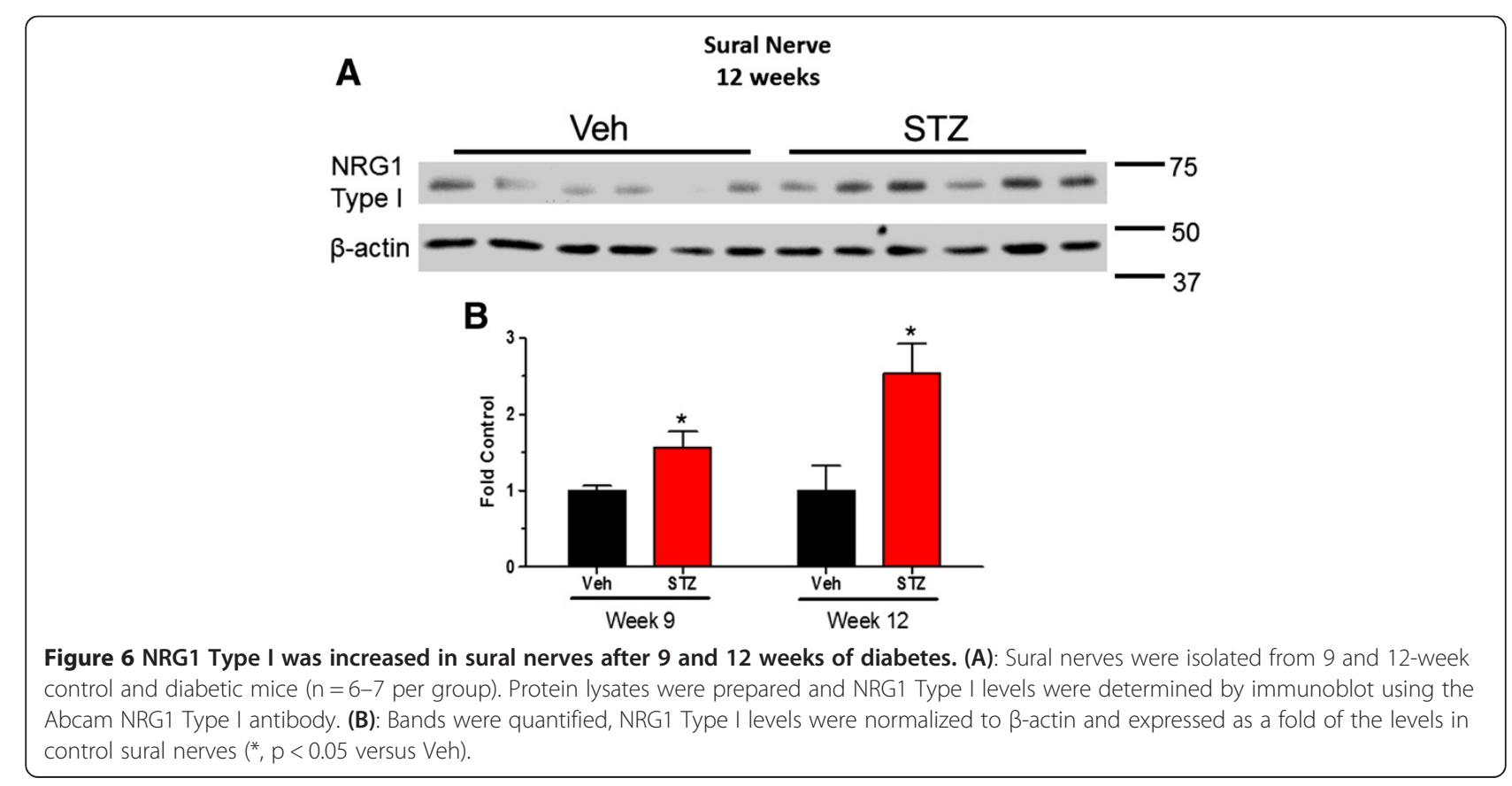



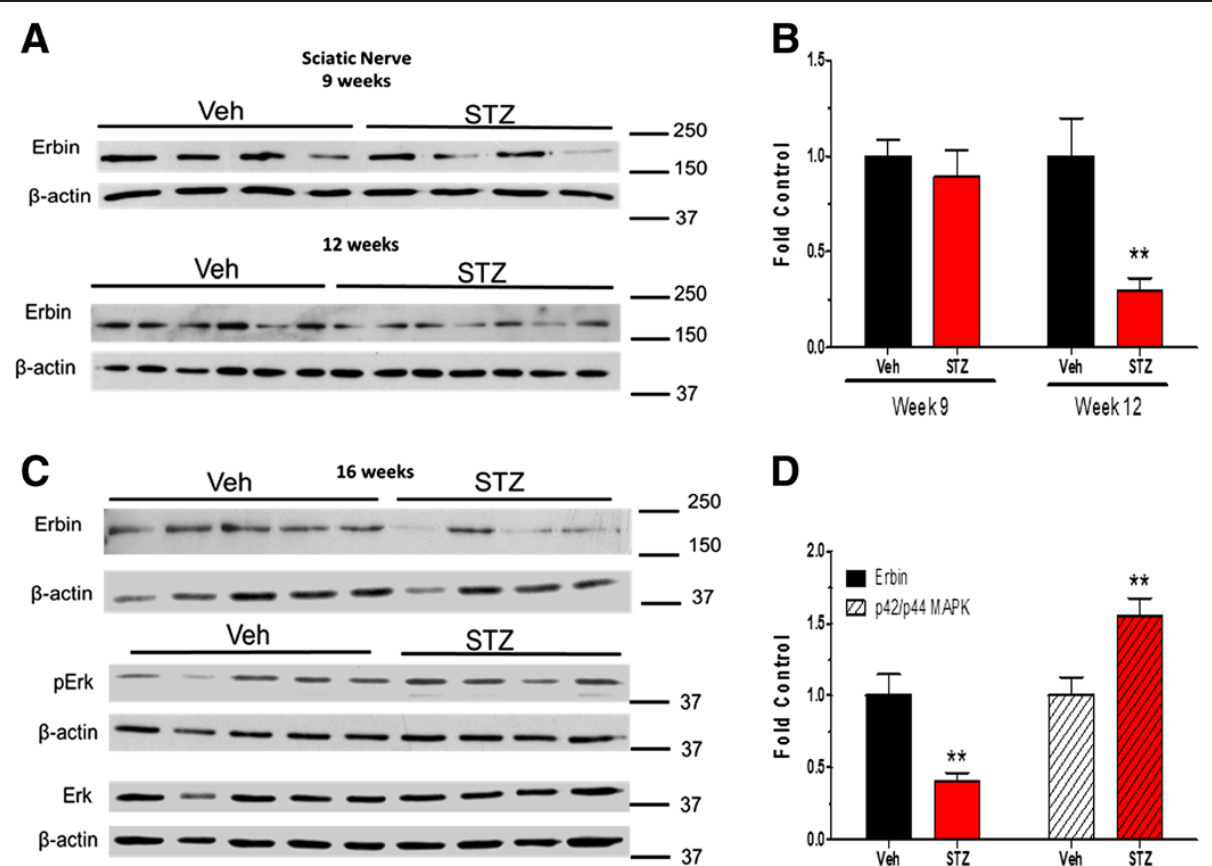

D

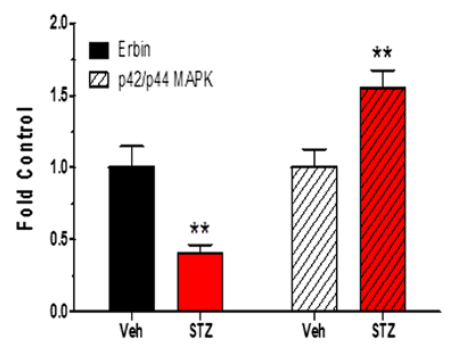

Figure 7 Erbin was decreased and p42/p44 MAPK activity enhanced in diabetic sciatic nerve. Sciatic nerves were isolated from 9,12 (A) and 16-week (C) control and diabetic mice ( $n=8-9$ per group). Protein lysates were prepared and Erbin levels and p42/p44 MAPK (pErk) levels were determined by immunoblot. Quantification demonstrated a significant decrease of Erbin levels (B) and an increase in p42/p44 MAPK activation at 16 weeks (D). (*, $p<0.05$ versus Veh; ${ }^{* *}, p<0.01$ versus Veh).

process NRG1. NRG1 Type III processed by BACE1 produces a membrane-anchored, N-terminal fragment that promotes myelination [30], whereas TACE cleaves NRG1 Type III in the EGF-like domain and inactivates it, resulting in hypomyelination [31]. BACE cleavage also produces the $\mathrm{C}$-terminal fragment which can be degraded by $\gamma$-secretase. Though the C-terminal fragment is not necessary for myelination [32], it may be translocated to the nucleus of neurons and can repress apoptosis and promote survival [33]. Diabetes-induced changes in the expression of NRG1 Type III produced by BACE cleavage were verified using two antibodies which targeted either the CRD located within the $\sim 75 \mathrm{kDa} N$-terminal fragment or an epitope within the C-terminal fragment. Interestingly, we did not consistently observe the presence of unprocessed NRG1 Type III in nerves from either control or diabetic mice. This suggests that the decrease in the expression of the $\mathrm{N}$ - and C-terminal fragments of NRG1 Type III were not due to a decrease in proteolytic processing. Thus, changes in the rate of transcription and/or translation may contribute to the decrease in NRG1 Type III. However, we also observed an increase in NRG1 Type I in diabetic sural nerve. Since both isoforms are the product of alternative splicing of a single transcript, it is possible that diabetes-induced changes in mRNA processing may alter the isoform expression pattern. In this regard, diabetes has been shown to alter expression of transcriptional variants of the Slo gene that may contribute to erectile dysfunction [34]. However, diabetes may have tissue specific effects on the expression of NRG1 isoforms. For example, the levels of NRG1 Type I were decreased in diabetic rats with cardiomyopathy [35] and impaired signaling through the NRG1/Erb B cassette may contribute to the pathogenesis of diabetic cardiomyopathy, increasing susceptibility to heart failure [36].

The degeneration of sensory neurons in DPN is clearly associated with an alteration in neurotrophic support and disrupted NRG-1/Erb B2 signaling, presumably in SCs, may be interconnected with altered neurotrophism. BDNF is released from SCs, is decreased in diabetic rats [37] and treatment with BDNF prevented nerve conduction slowing and damage to large motor fibers [38]. A clear relationship exists between BDNF and NRG1 signaling since BDNF can also stimulate the secretion of soluble forms of NRG1 [39] whereas transgenic inhibition of endogenous Erb B2 via expression of a dominant-negative Erb B4 in non-myelinating SCs was sufficient to decrease the expression of BDNF [14]. Though it remains unclear whether changes in BDNF levels may have contributed to the altered expression of NRG1 isoforms observed in diabetic nerve in the current study, elucidating the effect of diabetes on the activity of neurotrophins and neuregulins in dedifferentiating and regenerating SCs may provide fundamental insight into the potential for pharmacologically regulating Erb B2 signaling at specific disease stages to improve nerve function. Lastly, recent data also 


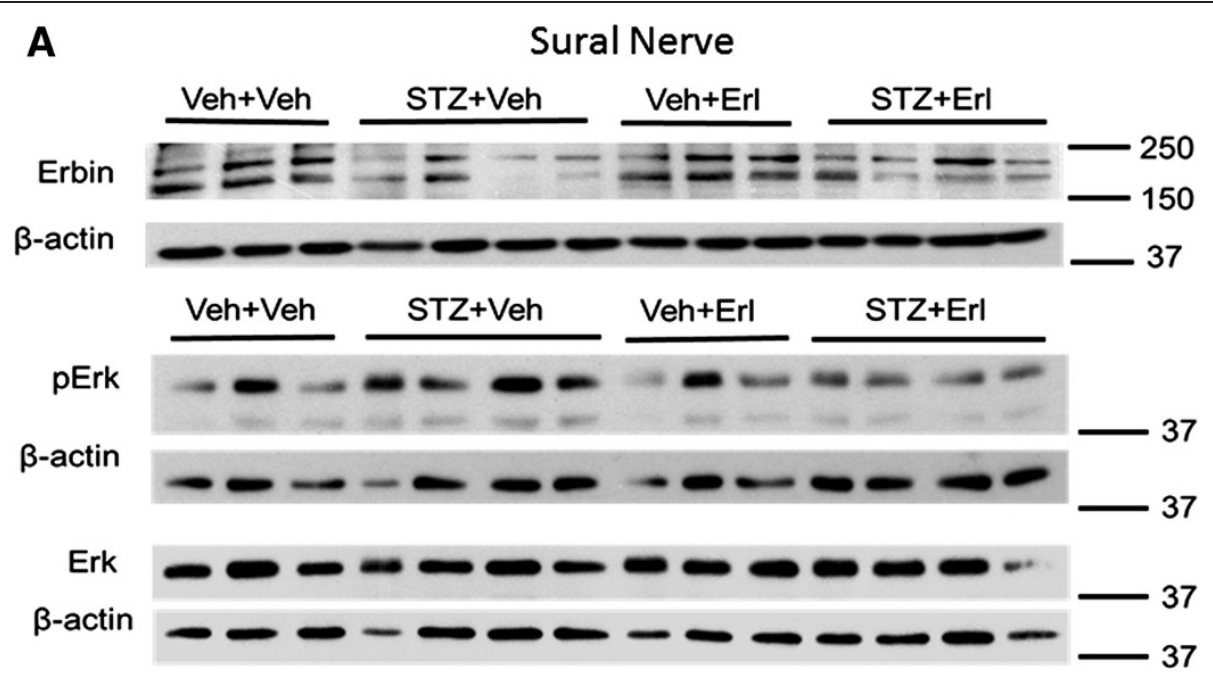

B

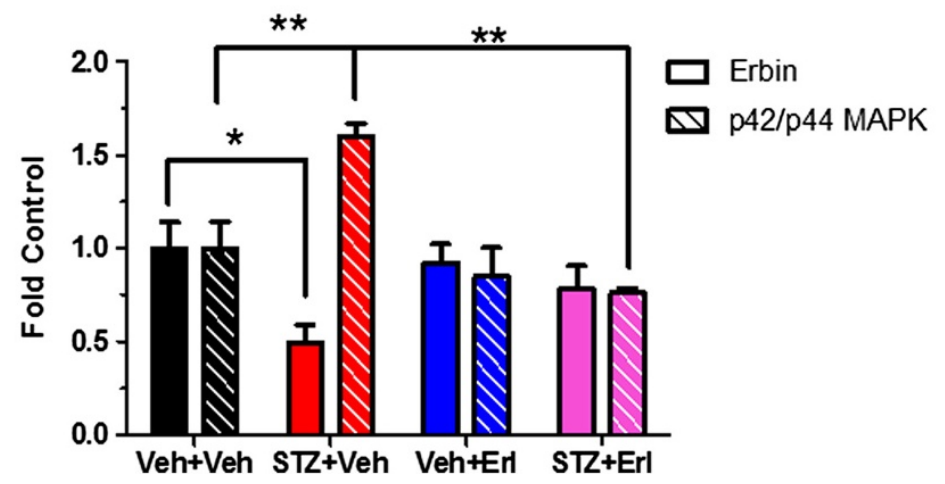

Figure 8 Inhibition of Erb B2 with erlotinib suppressed diabetes-induced p42/p44 MAPK pathway activation in sural nerves. (A): Sural nerves were isolated from vehicle or erlotinib-treated control and diabetic mice ( $n=3-4$ per group) at week 21. Protein lysates were prepared and erbin levels and p42/p44 MAPK (pErk) levels were determined by immunoblot. (B): Quantification demonstrated a significant decrease of erbin and an increase in p42/p44 MAPK activation in vehicle-treated diabetic mice. Erlotinib treatment suppressed p42/p44 MAPK activation in erlotinib-treated diabetic mice $\left({ }^{*}, \mathrm{p}<0.05 ;{ }^{* *}, \mathrm{p}<0.01\right)$.

suggests that axonal expression of NRG1 Type III can negatively regulate the expression of SC-derived NRG1 Type I [40]. Although these results were obtained in the context of a decrease in NRG1 Type III due to axonal loss following nerve crush, axonal loss is not a hallmark of the rather early stage of DPN modeled in our study. Thus, a diabetes-induced alteration in the expression of NRG1 Type III without frank axonal loss may be sufficient to promote the expression of NRG1 Type I. However, additional work is required to determine if the negative regulation of NRG1 Type I expression by axonal NRG1 Type III may be recapitulated following a peripheral nerve injury that is solely metabolic.

Erbin functions as an adapter protein that binds to Erb B2 and it plays an important role in myelination since erbin knockout mice have a decrease in Erb B2 levels and NRG1-induced myelination [20,41]. Erbin also serves as a negative regulator of $\mathrm{p} 42 / \mathrm{p} 44$ MAPK signaling by disrupting the interaction between Ras and Raf [28,42]. Consistent with this relationship, diabetes decreased erbin levels in sciatic and sural nerve and this correlated with an increase in the activity of $\mathrm{p} 42 / \mathrm{p} 44$ MAPK. Although erlotinib treatment inhibited the activation of $\mathrm{p} 42 / \mathrm{p} 44$ MAPK in sural nerve, without increasing erbin expression, it is not possible to directly link this change in MAPK activity to the improved sensory endpoints following erlotinib treatment since the drug would be expected to blunt all signaling through Erb B2. Though activation of the MAPK pathway has been associated with demyelination [18,43], myelin loss is not a hallmark of DPN in rodent models. Therefore, the activation of this pathway is either of insufficient magnitude and duration to promote demyelination in rodent nerve or contributes to other aspects of DPN. However, the contribution of $\mathrm{p} 42 / \mathrm{p} 44$ MAPK activity to DPN in both rodent and human DPN is unclear and 
these enzymes showed variable activation in sural nerve biopsies obtained from diabetic patients undergoing amputations [44].

\section{Conclusions}

In summary, our data support that diabetes may alter Erb B2 signaling in peripheral nerve by altering the balance in NRG1 isoform expression and decreasing the expression of erbin, an adapter protein that can function as a negative regulator of $\mathrm{p} 42 / \mathrm{p} 44$ MAPK signaling via Erb B2. Though a limitation of our study is that we can not ascertain if changes in NRG1 isoforms are necessary or sufficient to contribute to DPN, altered signaling through the NRG1-Erb B2 ligand/receptor pair may contribute to dysfunction of both myelinated and unmyelinated fibers since diabetic mice treated with erlotinib exhibited an improvement in both mechanical and thermal sensitivity. Given the complex role of neuregulins in controlling both myelination and demyelination, these data suggest that an altered neuregulinism may contribute to myelin pathologies that develop in human DPN.

\section{Methods}

\section{Reagents and antibodies}

Streptozotocin (STZ) was obtained from Sigma-Aldrich (St. Louis, MO). N-(3-ethynylphenyl)-6,7-bis(2-methoxyethoxy)4-quinazolinamine (erlotinib) was provided by OSI Pharmaceuticals (Melville, NY). NRG1 Type III polyclonal antibody was produced in rabbits by Chi Scientific (Maynard, MA) using the CIAGLKWVFVDKIFEYDSPTHL peptide of NRG1 Type III as the immunizing antigen [12]. CIAGLKWVFVDKIFEYDSPTHL peptide was synthesized by Selleck Chemicals (Houston, TX). A commercial NRG1 Type III antibody (SMDF C-16) and horseradish peroxidase-conjugated secondary antibodies were obtained from Santa Cruz Biotechnology (Santa Cruz, CA). NRG1 Type I (ab27303) and erbin (ab55930) antibodies were purchased from Abcam (Cambridge, MA). Other antibodies and their sources were: $\beta$-actin antibody (\#691002, MP Biomedicals, Solon, OH); phospho-MAPK 1/2 (\#9101) and total MAPK 1/2 (\#9102) antibodies (Cell Signaling, Boston, MA).

\section{Induction of diabetes and drug treatment}

Swiss Webster mice were obtained from Harlan Laboratories (Indianapolis, IN) and eight-week old mice were rendered diabetic with freshly prepared STZ given by intraperitoneal injection at $100 \mathrm{mg} / \mathrm{kg}$ for two consecutive days. One week after the last injection, mice were fasted for $6 \mathrm{hrs}$ and blood was obtained from the tail vein. Mice with a fasting blood glucose $\geq 290 \mathrm{mg} / \mathrm{dl}(16 \mathrm{mmol} / \mathrm{l})$ were deemed diabetic (One-Touch Ultra glucometer). All animals were maintained on a 12-h light/dark cycle with ad libitum access to water and Purina diet 5001 rodent chow.
To inhibit Erb B2, erlotinib was dissolved in $0.1 \mathrm{mM}$ Captisol and given at $25 \mathrm{mg} / \mathrm{kg}$ via intraperitoneal injection either once or twice per week. Final levels of fasting blood glucose and glycated hemoglobin (HbA1c, A1C $\mathrm{Now}^{+}$) were determined immediately before euthanizing the animals. All animal procedures were performed in accordance with protocols approved by the Institutional Animal Care and Use Committee and in compliance with standards and regulations for care and use of laboratory rodents set by the National Institutes of Health.

\section{Measure of sensory hypoalgesia and nerve conduction velocities (NCV)}

Mechanical and thermal sensitivity were measured as previously described using a Dynamic Plantar Aesthesiometer (Stoelting, Wood Dale, IL) and a Hargreaves Analgesiometer, respectively [26]. Withdrawal force (grams) and paw withdrawal latency (seconds) are the average of three to four trials taken from alternating feet with 5-min periods between testing. Motor and sensory nerve conduction velocities were assessed in deeply anesthetized mice as previously detailed [21]. Whole body (rectal probe) and near nerve temperatures (subcutaneous probe) of anesthetized mice were maintained at $36-37^{\circ} \mathrm{C}$ using a heating pad and heat lamp.

\section{Immunoblot analysis}

Peripheral nerves were homogenized in 0.2-ml lysis buffer $(50 \mathrm{mmol} / \mathrm{l}$ Tris-HCl, pH 7.5, $1 \mathrm{mmol} / \mathrm{l}$ EDTA, $1 \%$ Nonidet P-40, 0.5\% deoxycholate, $1 \mathrm{mmol} / \mathrm{l} \mathrm{Na}_{3} \mathrm{VO}_{4}$, $150 \mathrm{mmol} / \mathrm{l} \mathrm{NaCl}, 0.5 \mathrm{mmol} / \mathrm{l}$ sodium molybdate, 40 $\mathrm{mmol} / \mathrm{l} \mathrm{NaF}, 10 \mathrm{mmol} / \mathrm{l} \beta$-glycerophosphate, and $1 \mathrm{X}$ Complete Protease inhibitors) (Roche Diagnostics) with the aid of a Polytron fitted with a micro tissue tearor. Cell debris was sedimented at $10,000 \times g$ for $5 \mathrm{~min}$ at $4^{\circ} \mathrm{C}$, and the protein concentration of the supernatant was determined. Proteins were separated by SDS-PAGE and transferred onto nitrocellulose for immunoblot analyses. Immunoblots were quantified by densitometry with the aid of ImageJ software and the level of the proteins of interest was normalized to $\beta$-actin unless otherwise stated. Changes in protein expression are expressed as a percent of the control values.

\section{Peptide competition assay}

Sciatic nerves from untreated adult mice were homogenized in lysis buffer, proteins were separated by SDSPAGE and transferred onto nitrocellulose for immunoblot analyses. The various NRG1 antibodies were preabsorbed with buffer or the immunizing peptide at a 1:50, 1:100 or 1:200 mass ratio by incubation for $1 \mathrm{hr}$ at $25^{\circ} \mathrm{C}$ prior to their use for detecting NRG1 isoforms in the nerve lysate by immunoblot analysis. 


\section{Immunohistochemistry analysis}

The integument of the plantar surface of both hind paws was dissected and placed in Zamboni's fixative overnight. Tissues were then rinsed in PBS at $4^{\circ} \mathrm{C}$, cryoprotected in $30 \%$ sucrose overnight, embedded in OCT, frozen on dry ice, and stored at $-80^{\circ} \mathrm{C}$. Frozen tissues were sectioned at $30 \mu \mathrm{m}$, placed on Fisherbrand Superfrost Plus microscope slides and stored at $-80^{\circ} \mathrm{C}$. Immunohistochemistry was performed using the Vectastain Elite ABC-Peroxidase kit for rabbit IgG (Vector Laboratories, Burlingame, CA) and an anti PGP 9.5 antibody (AbD Serotec, Oxford, UK). Sections were then counterstained by NovaRED peroxidase substrate solution (Vector Laboratories, Burlingame, CA) and with hematoxylin. A total of twelve images per animal were captured on a Zeiss LSM 510 Meta confocal microscope for quantification. Single nerve fibers crossing the dermal/epidermal junction were counted from each image. The IENF density was calculated by the number of fibers counted divided by the length of the dermal/epidermal junction (fibers $/ \mathrm{mm}$ ).

\section{Statistical analysis}

Data are presented as means \pm SEM. After verifying equality of variance, differences between treatments were determined using a one-way or two-way ANOVA. Differences between group means were ascertained using Tukey's test.

\section{Abbreviations \\ BACE1: $\beta$-site amyloid precursor protein cleaving enzyme 1; BDNF: Brain derived-nerurotrophic factor; CRD: Cysteine-rich domain; DPN: Diabetic peripheral neuropathy; EGF: Epidermal growth factor; EGFR: Epidermal growth factor receptor; Erbin: Erb B2-interacting protein; \\ IENFD: Intraepidermal nerve fiber density: MAPK: Mitogen-activated protein kinase; MNCV: Motor nerve conduction velocity; NGF: Nerve growth factor; NRG1: Neuregulin-1; PGP 9.5: Protein gene product 9.5; SC: Schwann cell; SMDF: Sensory and motor neuron derived factor; SNCV: Sensory nerve conduction velocity; STZ: Streptozotocin; TACE: Tumor necrosis factor-a-converting enzyme.}

\section{Competing interests}

The authors declare that they have no competing interests.

\section{Authors' contributions}

PP performed the study and wrote the manuscript; RTD designed the research, wrote and edited the manuscript. Both authors read and approved the final manuscript.

\section{Acknowledgements}

This work was supported by grants from the Juvenile Diabetes Research Foundation and The National Institutes of Health [NS054847, NS075311 and DK095911]. We also thank OSI Pharmaceuticals for the generous supply of erlotinib.

Received: 10 July 2013 Accepted: 15 July 2013

Published: 17 July 2013

\section{References}

1. Callaghan BC, Cheng HT, Stables CL, Smith AL, Feldman EL: Diabetic neuropathy: clinical manifestations and current treatments. Lancet Neurol 2012, 11:521-534.
2. Zochodne DW: Diabetes mellitus and the peripheral nervous system: manifestations and mechanisms. Muscle Nerve 2007, 36:144-166.

3. Sinnreich M, Taylor BV, Dyck PJ: Diabetic neuropathies. Classification, clinical features, and pathophysiological basis. Neurologist 2005, 11:63-79.

4. Obrosova IG: Diabetes and the peripheral nerve. Biochim Biophys Acta 2009, 10:931-940.

5. Calcutt NA, Jolivalt CG, Fernyhough P: Growth factors as therapeutics for diabetic neuropathy. Curr Drug Targets 2008, 9:47-59.

6. Leinninger GM, Feldman EL: Insulin-like growth factors in the treatment of neurological disease. Endocrine Dev 2005, 9:135-159.

7. Liu X, Bates R, Yin DM, Shen C, Wang F, Su N, Kirov SA, Luo Y, Wang JZ, Xiong WC, Mei L: Specific regulation of NRG1 isoform expression by neuronal activity. J Neurosci 2011, 31:8491-8501.

8. Esper RM, Pankonin MS, Loeb JA: Neuregulins: Versatile growth and differentiation factors in nervous system development and human disease. Brain Res Brain Res Rev 2006, 51:161-175.

9. Grinspan JB, Marchionni MA, Reeves M, Coulaloglou M, Scherer SS: Axonal interactions regulate Schwann cell apoptosis in developing peripheral nerve: neuregulin receptors and the role of neuregulins. J Neurosci 1996 , 16:6107-6118

10. Nave KA, Salzer JL: Axonal regulation of myelination by neuregulin 1 . Curr Opin Neurobiol 2006, 16:492-500.

11. Taveggia C, Zanazzi G, Petrylak A, Yano H, Rosenbluth J, Einheber S, Xu X, Esper RM, Loeb JA, Shrager P, Chao MV, Falls DL, Role L, Salzer JL: Neuregulin-1 type III determines the ensheathment fate of axons. Neuron 2005, 47:681-694.

12. Michailov GV, Sereda MW, Brinkmann BG, Fischer TM, Haug B, Birchmeier C, Role L, Lai C, Schwab MH, Nave KA: Axonal neuregulin-1 regulates myelin sheath thickness. Science 2004, 304:700-703.

13. Chen S, Velardez MO, Warot X, Yu Z-X, Miller SJ, Cros D, Corfas G: Neuregulin 1-ErbB signaling is necessary for normal myelination and sensory function. J Neurosci 2006, 26:3079-3086.

14. Chen S, Rio C, Ji RR, Dikkes P, Coggeshall RE, Woolf CJ, Corfas G: Disruption of ErbB receptor signaling in adult non-myelinating Schwann cells causes progressive sensory loss. Nat Neurosci 2003, 6:1186-1193.

15. Guertin AD, Zhang DP, Mak KS, Alberta JA, Kim HA: Microanatomy of axon/ glial signaling during Wallerian degeneration. J Neurosci 2005, 25:3478-3487.

16. Zanazzi G, Einheber S, Westreich R, Hannocks MJ, Bedell-Hogan D, Marchionni MA, Salzer JL: Glial growth factor/neuregulin inhibits Schwann cell myelination and induces demyelination. J Cell Biol 2001, 152:1289-1299.

17. Syed N, Reddy K, Yang DP, Taveggia C, Salzer JL, Maurel P, Kim HA: Soluble neuregulin-1 has bifunctional, concentration-dependent effects on schwann cell myelination. J Neurosci 2010, 30:6122-6131.

18. Napoli I, Noon Luke A, Ribeiro S, Kerai Ajay P, Parrinello S, Rosenberg Laura H, Collins Melissa J, Harrisingh Marie C, White lan J, Woodhoo A, Lloyd Alison C: A central role for the ERK-signaling pathway in controlling Schwann cell plasticity and peripheral nerve regeneration in vivo. Neuron 2012, 73:729-742.

19. Borg JP, Marchetto S, Le Bivic A, Ollendorff V, Jaulin-Bastard F, Saito $H$, Fournier E, Adelaide J, Margolis B, Birnbaum D: ERBIN: a basolateral PDZ protein that interacts with the mammalian ERBB2/HER2 receptor. Nat Cell Biol 2000, 2:407-414

20. Tao Y, Dai P, Liu Y, Marchetto S, Xiong WC, Borg JP, Mei L: Erbin regulates NRG1 signaling and myelination. Proc Natl Acad Sci 2009, 106:9477-9482.

21. McGuire JF, Rouen S, Siegfreid E, Wright DE, Dobrowsky RT: Caveolin-1 and altered neuregulin signaling contribute to the pathophysiological progression of diabetic peripheral neuropathy. Diabetes 2009, 58:2677-2686.

22. Kennedy JM, Zochodne DW: Experimental diabetic neuropathy with spontaneous recovery: is there irreparable damage? Diabetes 2005, 54:830-837.

23. Malik RA, Veves A, Tesfaye S, Smith G, Cameron N, Zochodne D, Lauria G, on behalf of The Toronto Consensus Panel on Diabetic N: Small fibre neuropathy: role in the diagnosis of diabetic sensorimotor polyneuropathy. Diab Metab Res Rev 2011, 27:678-684.

24. Johnson MS, Ryals JM, Wright DE: Early loss of peptidergic intraepidermal nerve fibers in an STZ-induced mouse model of insensate diabetic neuropathy. Pain 2008, 140:35-47.

25. Beiswenger KK, Calcutt NA, Mizisin AP: Dissociation of thermal hypoalgesia and epidermal denervation in streptozotocin-diabetic mice. Neurosci Lett 2008, 442:267-272. 
26. Urban MJ, Pan P, Farmer KL, Zhao H, Blagg BS, Dobrowsky RT: Modulating molecular chaperones improves sensory fiber recovery and mitochondrial function in diabetic peripheral neuropathy. Exp Neurol 2012, 235:388-396.

27. Wang JY, Miller SJ, Falls DL: The N-terminal region of neuregulin isoforms determines the accumulation of cell surface and released neuregulin ectodomain. J Biol Chem 2001, 276:2841-2851.

28. Huang $Y Z$, Zang M, Xiong WC, Luo Z, Mei L: Erbin suppresses the MAP kinase pathway. J Biol Chem 2003, 278:1108-1114.

29. Hinder LM, Vivekanandan-Giri A, McLean LL, Pennathur S, Feldman EL: Decreased glycolytic and tricarboxylic acid cycle intermediates coincide with peripheral nervous system oxidative stress in a murine model of type 2 diabetes. J Endocrinol 2013, 216:1-11.

30. Fleck D, Garratt AN, Haass C, Willem M: BACE1 dependent neuregulin processing: review. Curr Alzheimer Res 2012, 9:178-183.

31. La Marca R, Cerri F, Horiuchi K, Bachi A, Feltri ML, Wrabetz L, Blobel CP, Quattrini A, Salzer JL, Taveggia C: TACE (ADAM17) inhibits Schwann cell myelination. Nat Neurosci 2011, 14:857-865.

32. Velanac V, Unterbarnscheidt T, Hinrichs W, Gummert MN, Fischer TM, Rossner MJ, Trimarco A, Brivio V, Taveggia C, Willem M, Haass C, Möbius W, Nave K-A, Schwab MH: Bace1 processing of NRG1 type III produces a myelin-inducing signal but is not essential for the stimulation of myelination. Glia 2012, 60:203-217.

33. Bao J, Wolpowitz D, Role LW, Talmage DA: Back signaling by the Nrg-1 intracellular domain. J Cell Biol 2003, 161:1133-1141.

34. Davies KP, Zhao W, Tar M, Fiqueroa JC, Desai P, Verselis VK, Kronengold J, Wang H-Z, Melman A, Christ GJ: Diabetes-induced changes in the alternative splicing of the Slo gene in corporal tissue. Eur Urol 2007, 52:1229-1237.

35. Gui C, Zhu L, Hu M, Lei L, Long Q: Neuregulin-1/ErbB signaling is impaired in the rat model of diabetic cardiomyopathy. Cardiovasc Path 2012, 21:414-420.

36. Odiete O, Konik EA, Sawyer DB, Hill MF: Type 1 diabetes mellitus abrogates compensatory augmentation of myocardial neuregulin-1 beta/ErbB in response to myocardial infarction resulting in worsening heart failure. Cardiovasc Diabetol 2013, 12:52.

37. Mizisin AP, DiStefano PS, Liu X, Garrett DN, Tonra JR: Decreased accumulation of endogenous brain-derived neurotrophic factor against constricting sciatic nerve ligatures in streptozotocin-diabetic and galactose-fed rats. Neurosci Lett 1999, 263:149-152.

38. Mizisin AP, Bache M, DiStefano PS, Acheson A, Lindsay RM, Calcutt NA: BDNF attenuates functional and structural disorders in nerves of galactose-fed rats. J Neuropathol Exp Neurol 1997, 56:1290-1301.

39. Ma Z, Wang J, Song F, Loeb JA: Critical period of axoglial signaling between neuregulin-1 and brain-derived neurotrophic factor required for early Schwann cell survival and differentiation. J Neurosci 2011, 31:9630-9640.

40. Stassart RM, Fledrich R, Velanac V, Brinkmann BG, Schwab MH, Meijer D, Sereda MW, Nave KA: A role for Schwann cell-derived neuregulin-1 in remyelination. Nat Neurosci 2013, 16:48-54.

41. Liang C, Tao Y, Shen C, Tan Z, Xiong WC, Mei L: Erbin is required for myelination in regenerated axons after injury. J Neurosci 2012, 32:15169-15180.

42. Rangwala R, Banine F, Borg JP, Sherman LS: Erbin regulates mitogenactivated protein (MAP) kinase activation and MAP kinase-dependent interactions between merlin and adherens junction protein complexes in Schwann cells. J Biol Chem 2005, 280:11790-11797.

43. Harrisingh MC, Perez-Nadales E, Parkinson DB, Malcolm DS, Mudge AW, Lloyd AC: The Ras/Raf/ERK signalling pathway drives Schwann cell dedifferentiation. EMBO J 2004, 23:3061-3071.

44. Purves TD, Middlemas A, Agthong SI, Jude EB, Boulton AJM, Fernyhough $P$, Tomlinson DR: A role for mitogen-activated protein kinases in the etiology of diabetic neuropathy. FASEB J 2001, 15:2508-2514.

doi:10.1186/2051-5960-1-39

Cite this article as: Pan and Dobrowsky: Differential expression of neuregulin-1 isoforms and downregulation of erbin are associated with Erb B2 receptor activation in diabetic peripheral neuropathy. Acta Neuropathologica Communications 2013 1:39.

\section{Submit your next manuscript to BioMed Central and take full advantage of:}

- Convenient online submission

- Thorough peer review

- No space constraints or color figure charges

- Immediate publication on acceptance

- Inclusion in PubMed, CAS, Scopus and Google Scholar

- Research which is freely available for redistribution 